\title{
Validity and reproducibility testing of the Molar Incisor \\ Hypomineralisation (MIH) Index
}

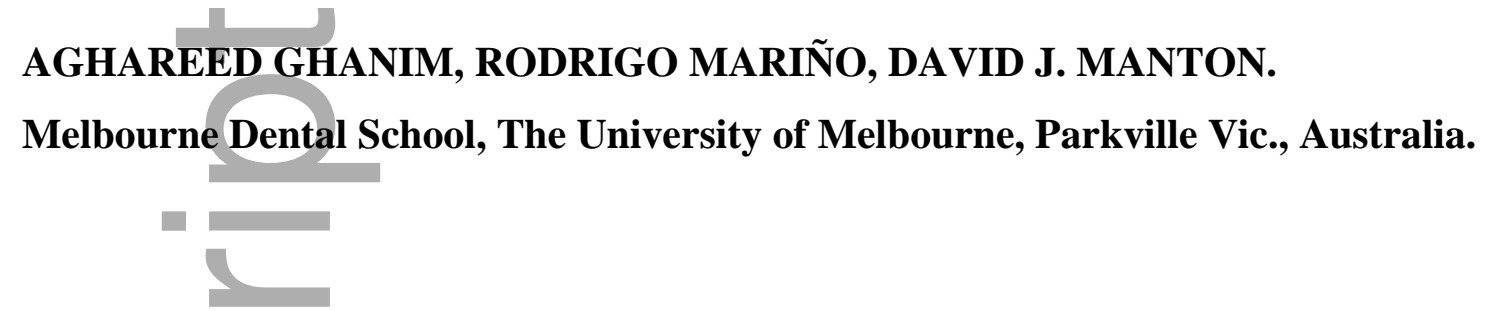

Running title: MIH index validation.

Number of words in abstract 198

Number of words in abstract and text including references and bullet points 4309

Authors

Dr Aghareed Ghanim: Clinical Senior Fellow, Melbourne Dental School, The University of Melbourne, Australia.

E-mail: aghareed.alqassab@unimelb.edu.au

Professor Rodrigo Mariño: Principal Research Fellow, Oral Health Cooperative Research Centre, Melbourne Dental School, The University of Melbourne, Australia.

E-mail: marino@unimelb.edu.au



Professor David Manton: Elsdon Storey Chair of Child Dental Health, Melbourne Dental School, The University of Melbourne, Australia.

E-mail:djmanton@unimelb.edu.au

This is the author manuscript accepted for publication and has undergone full peer review but has not been through the copyediting, typesetting, pagination and proofreading process, which may lead to differences between this version and the Version of Record. Please cite this article as doi: 10.1111/ipd.12433

This article is protected by copyright. All rights reserved 
Correspondence address: Dr Aghareed Ghanim

Melbourne Dental School

The University of Melbourne, Vic. 3010 Australia.

Ph: +61 (0)3 39341521

Fax: 061393411595

E-mail: aghareed.alqassab@unimelb.edu.au

\section{Why this paper is important to paediatric dentists}

- Molar-Incisor Hypomineralisation (MIH) has many negative impacts on patients and presents diagnostic and treatment challenges to clinicians. A new assessment tool for measuring MIH/HSPM lesions was developed consistent with existing judgment criteria to grade the clinical conditions, amount of tooth surface area affected and other enamel defects.

- The study provides initial evidence of the reproducibility and validity of the MIH index to be considered a valid and reliable tool for MIH/HSPM assessment in clinical practice and large epidemiological studies.

- Future research using this index would provide additional information aimed at determining worldwide MIH prevalence.

\section{Authors' contributions}

AG and RM contributed to the conception of the manuscript. AG and RM performed the statistical analysis, critical evaluation and writing up of the manuscript. AG, RM and DM subsequently revised the drafts. All authors read and approved the final manuscript.

\section{Acknowledgements}

Not applicable

This article is protected by copyright. All rights reserved 


\section{Conflict of interest}

The authors have no conflict of interests for publication of this manuscript. All authors have made substantive contribution to this study and/or manuscript, and all have reviewed the final paper prior to its submission.

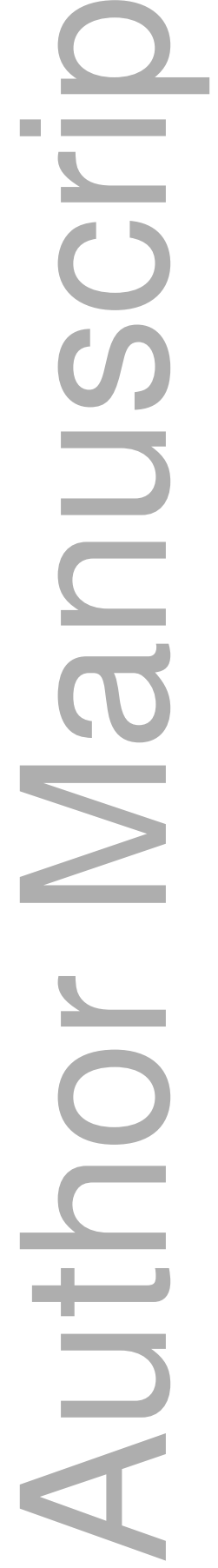


DR. AGHAREED GHANIM (Orcid ID : 0000-0001-7746-1516)

PROF. DAVID JOHN MANTON (Orcid ID : 0000-0002-4570-0620)

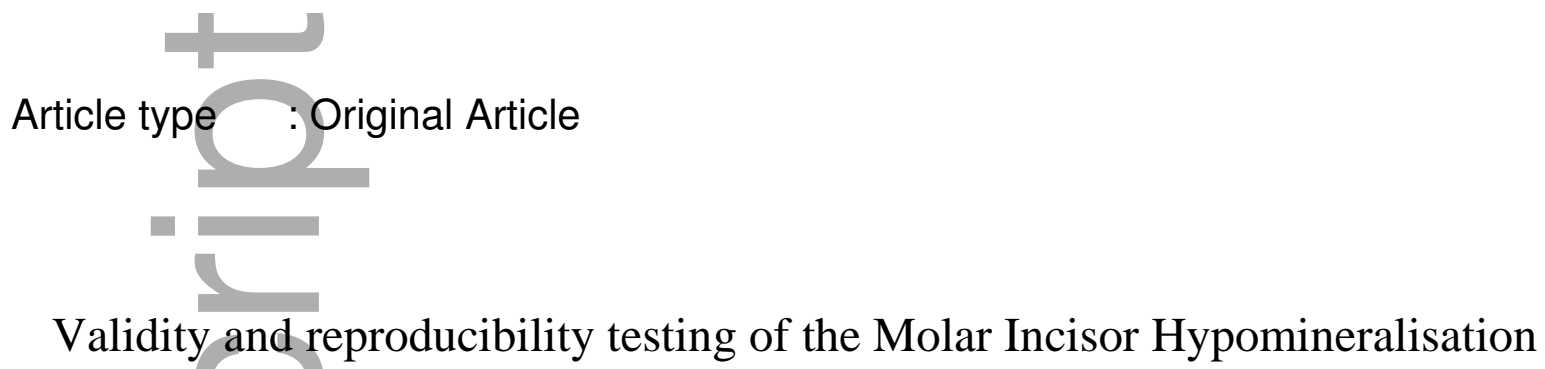
(MIH) Index

\section{Summary}

Background. To address challenges presented to clinicians to diagnose and treat MolarIncisor Hypomineralisation (MIH), a new assessment tool was created.

Aim. To explore the reproducibility and validity of a new instrument developed to assess MIH.

Methods. Inter-rater and test-retest reliability were examined using Cronbach's alpha. Thirtyfive oral health professionals scored clinical photographs for the presence/absence of MIH and other enamel defects using the new index. Face, content, and construct validity was assessed. A panel of six experienced researchers assessed face validity. Construct validity was determined by examining hypothesised associations with clinical variables reported to change concurrently or as modifiers of the MIH outcome. Sensitivity, specificity, Positive Predictive Value/PPV, Negative Predictive Value/NPV were also assessed.

Results: The index reflected the hypothesized associations regarding the presence, prevalence, and severity of $\mathrm{MIH}$; demonstrating construct validity. Researchers indicated that codes and definitions were clear and suitable for international use. The index showed satisfactory scores of sensitivity/specificity, and PPV/NPV. Examiners achieved "Substantial" to "Almost perfect" levels of agreement in clinical presentation and lesion extension. 
Conclusions. The tested MIH index had reasonably solid properties providing confidence that it is a reliable and valid instrument for use in population-based and clinical screenings for diagnosis of $\mathrm{MIH}$ and other enamel defects.

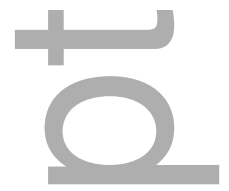

\section{Introduction}

Demarcated enamel hypomineralisation is defined as a demarcated qualitative defect of the enamel visually identified as an abnormality in translucency; denominated as Molar Incisor Hypomineralisation (MIH) when affecting the first permanent molars and often the permanent incisors and systemically derived; a globally prevalent condition (approximately $14 \%)^{1,2}$. The defining clinical features of MIH are demarcated opacities with clear, distinct borders with the adjacent enamel. The opacities range from white to yellow/brown in appearance, and in lesions with severe mineral deficit, rapid progression to post-eruptive enamel breakdown (PEB) can occur ${ }^{1}$. These qualitative defects of enamel characteristically present asymmetrically on one or more of the permanent first molars, with or without the involvement of the permanent incisors. The presence of these defects on the permanent incisors suggests a more severe form of the condition ${ }^{3}$. This notion extends to the primary dentition, where the presence of hypomineralisation in primary teeth, commonly referenced in the literature as Hypomineralised Second Primary Molars (HSPM) ${ }^{4}$, is reported as a predictive factor for MIH. It is reported that people at increased risk of dental caries affected by MIH or HSPM have worse outcomes regarding carious lesion development, and the condition negatively impacts their quality of life $\mathrm{e}^{5,6}$.

There has been growing interest and research in MIH since its formal recognition in $2001^{1}$. On the other hand, there are no standard criteria to identify and classify MIH lesions. Most previous studies utilised either the European Academy of Paediatric Dentistry (EAPD) judgment criteria, modified index of developmental defects of enamel (mDDE), and individual criteria to identify and illustrate the cases of MIH and $\mathrm{HSPM}^{7}$. MIH prevalence rates vary from $2.4 \%$ to $44 \%$; highlighting the vast disparity in the reported prevalence rates across the world, the result of a lack of internationally utilised and reliable criteria for diagnosing MIH and $\mathrm{HSPM}^{7,8}$. For HSPM, prevalence ranges between $0 \%$ and $21.8 \%{ }^{7}$. 
Recently, a new index has been developed to be used for the diagnosis of MIH/HSPM ${ }^{9}$. Compared with the previous criteria and indices, the MIH/HSPM index combines principles of the EAPD judgment criteria and mDDE index in order to grade the clinical status, amount of tooth surface area affected and other enamel defects comparable to $\mathrm{MIH}^{9}$. The new index potentially minimises misdiagnosis of MIH/HSPM, is available for a wide range of ages to explore the prevalence of MIH/HSPM and determines the prevalence changes over time. A training module was developed to assist researchers in implementing the index in a standardised manner ${ }^{10}$.

Several researchers are using/have used the index ${ }^{11-16}$, but its properties, in terms of validity and reproducibility, are yet to be tested. As with any new index, the MIH/HSPM index needs to demonstrate adequate reproducibility (i.e. reliability) and validity to be used for clinical assessment. The aim of the present study is to assess the validity and reproducibility of the new index of MIH/HSPM. The new index would allow better comparison of MIH/HSPM prevalence data between different geographical regions ${ }^{7,10}$.

\section{Materials and Methods}

The present study focuses on real data from two previous prevalence studies to explore the reproducibility and validity of the index in different age-groups and different populations ${ }^{11,14}$. In the first study, the prevalence of MIH among 424 randomly selected children (8-12 years) in Sabah, East Malaysia was $16.0 \%{ }^{14}$. In the second study, the prevalence of HSPM among 623 children (3-5 years) randomly selected in Melbourne, Victoria, Australia was $14.1 \%$. Based on these two studies, the instrument's face, content, and construct validity, as well as internal consistency, inter- and intra-examiner and test-re-test reliabilities were examined.

Description of the index codes is provided (Table 1). To assess the psychometric properties of the newly developed index, a research protocol was designed to test validity (construct, content and face) and reliability (inter-rater and test-retest). Validity of a measurement is usually evaluated as how an index's score accurately represents the theoretical construct under study ${ }^{17,18}$. As there is no standard criterion measure for MIH to be used as comparator, the construct validity approach was relied on to test validity of the index. This approach involves the examination of hypothesised associations with "concepts concerning the phenomenon under study"19. Therefore, construct validity was assessed through examination of clinical variables with hypothesized associations that have been reported to regarding the presence, prevalence, and severity of MIH/HSPM. Socio- 
demographic and general health variables were not included in this analysis as there is no universal agreement on their association with presence, and severity of $\mathrm{MIH}^{20}$. Six features have been reasonably delineated in the literature regarding $\mathrm{MIH}^{7,8,21}$. Directions of the measures predicting presence and severity of MIH are in the Results section to save space. Significant effects were examined by one-way analysis of variance (ANOVA). To further explore and interpret MIH scores, a significant ANOVA was followed by post-hoc tests (Tukey's Honestly Significant Differences) to determine which groups were different score on each of the independent variables examined. To better understand the relationship between the combination of clinical variables and $\mathrm{MIH}$, the probability was explored utilising a logistic regression analysis (LRA). Level of significance was set at a p-value of 0.05. Before the analysis, data were screened for violation of the assumptions underlying parametric methods. Analyses were carried out using IBM SPSS Statistics (24.0, IBM Corp, NY, USA).

Please insert Table 1 about here

Additionally, two other types of validity were addressed in the present analysis; content and face validities. Content validity explores how well the instrument developed incorporates the domain of the phenomenon under study ${ }^{19,22}$. Face validity uses expert agreement on whether the Index represented those characteristics and features of MIH/HSPM well ${ }^{16}$.

To test content validity, six experienced researchers assessed if the codes and definitions included appropriately reflected what should be reported and whether the different clinical presentations of MIH/HSPM were well captured in these codes and definitions. Thus, face and content validities ensure the inclusion of a comprehensive range of items that appropriately represented MIH/HSPM. As there is no statistical method to test whether the instrument accurately covers the content of a measure, the content and face validity will be presented through expert judgment ${ }^{22}$. A convenience sample of six world MIH experienced researchers, who were not part of the development of the MIH/HSPM index, provided their opinion on content of the index. Selection of the panel required the identifying of people from different backgrounds who were knowledgeable in the area as evidenced by publication in peer reviewed journals and were interested in the subject to be assessed. The panel was required to review the instrument's codes and definitions of the clinical status of enamel defects and to indicate by Yes or No to whether they appropriately reflected what is reported and whether all the different clinical presentations of MIH/HSPM were well captured in these codes and definitions. 
Reliability presents the consistency or repeatability of a measure, which is used to estimate the stability of measures used at different times or by different examiners ${ }^{23,24}$. Testretest reliability can be used to measure the instrument's stability over time, which indicates that the results of the test should be consistent each time it is used ${ }^{23,24}$. Inter-rater reliability aims to establish the degree of agreement by using the instrument amongst different examiners $^{23,24}$.

Inter-rater and test-retest reliability were assessed using a convenience sample of 35 oral health care providers (dentists and oral therapists) who reviewed clinical photographs collected from patients who were previously examined for the presence/absence of tooth development defects of enamel. The levels of inter-examiner and test-retest reliability were explored using the Kappa statistic ${ }^{25,26}$. Further, a valid index should present satisfactory scores of sensitivity, specificity, positive predictive value (PPV), and negative predictive value (NPV) ${ }^{27}$. Therefore, they were calculated with the data provided by the 35 oral health care providers using MEDCALC $^{\circledR}$ (12.0, MedCalc Software, Ostend, Belgium) to assess whether this index could be used to diagnose of MIH/HSPM affected teeth correctly ${ }^{28}$.

\section{Results}

\section{Construct validity}

\section{Presence and severity of MIH in permanent dentition}

a) Number of affected teeth and severity of lesion. [Assumption: When using the new index, individuals with more severe lesions will have more teeth involved.] ${ }^{3,29}$. Findings: Of 424 participants in Study One, 347 were enamel defect-free (i.e., no signs of MIH) in their first permanent molars and incisors. Of those participants who had first permanent molars and/or incisors with defects $(\mathrm{n}=77), 31(40.3 \%)$ had one permanent tooth affected; another 18 (23.4\%) had two teeth involved; twelve (15.6\%) participants had three teeth affected; and another ten $(13.0 \%)$ had four teeth affected. The remaining six participants $(7.8 \%)$ had between five and eight teeth affected, as a result the hypothesis was confirmed.

Severity of MIH was associated with the number of teeth with MIH experience ( $\mathrm{p}<$ $0.01)$; participants with opacities only had a lower mean number of teeth involved $(1.9 \pm 1.2$ teeth) compared to those with severe lesions (i.e., PEB, atypical restoration, atypical, extraction) cases of MIH (3.1 \pm 2.0 teeth). 
b) Type of lesions. [Assumption: When using the new index, the most common presentation will be white opacities followed by brown lesions and PEB.] ${ }^{21}$. Findings: Of the MIH lesions present $(n=178)$, the majority $(118 ; 66.3 \%)$ were white; $19.7 \%(n=35)$ were brown and the remaining $14.0 \%(\mathrm{n}=25)$ had $\mathrm{PEB}$, and therefore the hypothesis was confirmed.

c) Permanent Incisors involvement. [Assumption: Those who had increased involvement of first molars will also have increased likelihood of permanent incisor involvement.] ${ }^{21}$. Findings: Those with first molars affected with MIH were 2.4 times as likely to also have their permanent incisors also affected with MIH (Adjusted OR=2.40; 95\% CI 1.78 to 3.19; (p $<0.0001)$ which confirms the hypothesis.

d) Permanent Incisors enamel breakdown. [Assumption: When using the new index, PEB in permanent incisors should be rare. ${ }^{21}$. Findings: Although thirteen permanent incisors were affected with MIH, none had PEB, in that way the hypothesis was confirmed.

e) Number of affected teeth and dental caries experience. [Assumption: Children with more teeth affected with MIH will tend to have higher caries prevalence compared to MIH free children. A positive correlation was assumed. $]^{21}$. Findings: A higher number of teeth with MIH was positively correlated with dental caries experience $(\mathrm{p}<0.0001)$, and accordingly the hypothesis was confirmed.

f) $\mathrm{MIH}$ severity and number of teeth affected with dental caries. [Assumption: Severity of MIH will be positively correlated with dental caries experience in permanent dentition, compared to MIH free children] $]^{21}$. Findings: Severity of MIH was associated with the number of teeth with dental caries experience $(\mathrm{p}<0.0001)$. MIH-free children had a lower mean DMFT compared to any other level of MIH severity. Those with more severe MIH had significantly higher mean DMFT scores compared to those with demarcated opacity lesions, thereby the hypothesis was confirmed. (See Table 2).

Please insert Table 2 about here

g) Extension of MIH defect and dental caries experience. [Assumption: Independent of MIH severity, extension of MIH will be positively correlated with dental caries experience in permanent dentition $]^{21}$. Findings: Extension of the MIH defect was associated with the number of teeth with dental caries experience $(\mathrm{p}<0.0001)$. MIH-free children had a lower mean DMFT compared to those with MIH defects ( 0.20 vs. 3.32). In the same way, those with MIH extension of less than one-third of the tooth surface had a significantly lower mean DMFT compared to those with an MIH defect that extended beyond one-third of the tooth 
surface. However, those with an extension between one-third and two-thirds of the crown showed no statistically significant difference when compared to those with MIH defects extending beyond two-thirds of the crown, as a result the hypothesis was confirmed (See Table 2).

\section{Presence and severity of HSPM in primary dentition}

h) Type of lesions. [Assumption: When using the new index, the most common presentation of HSPM will be white opacities followed by, brown lesions and last atypical restorations.] $]^{21}$. Findings: Of 623 participants in Study Two, 535 were enamel defect-free (i.e., no signs of HSPM). Of those participants with defects $(n=88)$, the majority $(51.1 \%)$ had white defects, another $46.6 \%$ had brown lesions and of the remaining 2.2\%, one had PEB and one was missing due to HSPM, therefore the hypothesis was confirmed (See Table 3).

Please insert Table 3 about here

i) Relationship between MIH and HSPM. [Assumption: Children diagnosed with HSPM will be more likely to develop MIH than those who were HSPM-free. ${ }^{4,}{ }^{30}$. Findings: Children who had HSPM were almost 2.5 times more likely to have MIH in their permanent dentition [Adjusted OR $=2.45 ; 95 \%$ CI 1.20 to 5.01; $(\mathrm{p}<0.02)$ ], thus the hypothesis was confirmed.

\section{Face and content validity}

To ensure that the Index's represented well those characteristics and features of MIH/HSPM (i.e., content validity), six experienced researchers assessed if the included codes and definitions appropriately reflected what should be reported and whether the different clinical presentations of MIH/HSPM were well captured in these codes and definitions. Participating researchers unanimously indicated that codes and definitions were straightforward, clear, easy to apply, related to the MIH/HSPM and suitable for use internationally. Additionally, they indicated that the codes and definitions represented the condition, indicating that the index had content validity.

\section{Inter-examiner and test-retest reliabilities}

For test-retest reliability, most examiners achieved "Substantial" to "Almost perfect" levels of agreement according to Landis and Koch criteria, in both the clinical presentation (kappa range from 0.94 to 0.60 ) and for lesion extension (kappa range from 0.97 to 0.54$)^{25}$. In regard to inter-examiner reliability, "Substantial" to "Almost perfect" agreements were achieved by all examiners for both, the clinical presentation (kappa range from 0.97 to 0.61 ) and lesion extension (kappa range from 0.94 to 0.56 ) (See Table 4). 
Please insert Table 4 about here

\section{Sensitivity, specificity, PPV, and NPV}

Diagnosis of MIH vs DDE using the images when compared to the reference standard resulted in sensitivity of 98.0 (95\% CI: 97.1 - 98.6) and specificity of 98.0 (95\% CI: 96.2 98.9). Positive predictive values and negative predictive values were also high $(99.3 \%$ and $94.1 \%$, respectively) (See Table 5).

\section{Discussion}

Many studies have assessed the prevalence and presentation of MIH as fundamental information for clinical service provision and other purposes such as understanding aetiological and risk factors. These studies have also highlighted the need for improvements in the assessment and measurement of this condition ${ }^{8}$. The present results represent the validation of a new MIH tool which was developed consistent with current judgment criteria to grade the clinical statues, amount of tooth surface area affected and other enamel defects comparable to $\mathrm{MIH}^{9,31}$. The development of the $\mathrm{MIH}$ index as an assessment tool to measure $\mathrm{MIH}$ brings researchers in the area closer to filling this gap in the literature.

The validity and reproducibility of the MIH index were evaluated. Three types of validity were addressed; namely face, content, and construct validity. Face validity involved six experienced researchers looking at the MIH index items who agreed unanimously that the instrument was a theoretically valid measure of MIH. Content validity was assessed by ensuring the inclusion of a comprehensive range of items that appropriately represented the MIH clinically, which have been identified and defined in the literature. It included a comprehensive sample of items on the clinical aspects such as presence and severity of MIH in permanent and primary dentitions. The construct validity of the scale was established by using it successfully to test several hypotheses, derived from the literature, consistent with the MIH process and its clinical variables identified from previous studies on MIH. The hypotheses evaluated in the validity analysis were presented in the results section. During this validity analysis, MIH results confirmed our assumptions, thereby, providing evidence of the validity of the MIH index.

Regarding reliability, it constitutes an important issue in the use of any measurement method, and even more important when a new measurement is being developed ${ }^{23}$. Therefore, it was necessary to demonstrate that the instrument was repeatedly measuring what it was intended to measure ${ }^{23}$. In the present study, reliability was assessed as inter-examiner consistency and 
test-retest reliability, for the latter, the index demonstrated to have test-retest reliability. The magnitude of the kappa coefficient represents the proportion of agreement greater than that expected by chance. In the present study, with few exceptions, all reviewers reached or exceeded the minimum acceptable result (i.e., 0.70) ${ }^{23}$. As further evidence of its diagnostic accuracy, the MIH index, showed good sensitivity and specificity, which indicates that application of the index will correctly identify those with MIH and those with enamel defects other than MIH. Furthermore, the PPV, the probability of a child who receives a positive MIH result has the condition, was almost perfect, and the NPV (i.e., probability that the disease is not present when the test is negative) was also very high ${ }^{32}$.

The present study has strengths and weaknesses that must be considered. This evaluation focused on real data to explore the index's reproducibility and validity in different age groups and different populations. The samples had reasonable variation and fulfilled requirements for statistical analysis, including sufficient numbers of participants with various degrees of MIH. These two studies collected cross-sectional data only; and therefore, only tested clinical variables associated with MIH. Hypotheses around other risk factors (e.g., history of child/maternal illness, birth weight, alcohol consumption during pregnancy, etc.) could not be tested. Thus, if we want to unambiguously establish the scale's validity, longitudinal data should be collected to account for the complexities and multifactorial nature of MIH. Moreover, longitudinal studies are necessary to explore additional putative causal factors. Notwithstanding this, the present results support the continued use of the scale in research for measuring $\mathrm{MIH}$ and it provides the first evidence of its reproducibility and validity.

In view of the respective literature, the need to conduct more prevalence and etiological studies using standardized criteria was emphasized, otherwise MIH preventive and management programs may be difficult ${ }^{7,8,10,20,21}$. The present study was undertaken to develop an index that overcomes deficiencies of previous MIH criteria and indices and is simple to administer. The instrument was conceived, based on previous criteria (i.e., EAPD and $\mathrm{mDDE}$ ) with two forms ${ }^{9}$. Validation outcomes indicated promising results, and overall, the MIH index demonstrated adequate reliability and validity for group comparisons. Further development of this instrument would offer promising avenues for its use in population and clinical practice studies and would provide valuable information for policy makers designing preventive and management strategies, programs and services for the community.

There is no doubt that the instrument can be improved, but this preliminary investigation has confirmed, in a large sample of subjects, not only the different risk factors and clinical 
characteristics of $\mathrm{MIH}$, but that it is possible to measure using simple summated scales. In addition to further investigation of the reliability and the construct validity of the MIH index, directions for future research should, firstly, include comparable studies in other ethnic/racial groups, since its design allows - with some minor changes in the wording of items - crossvalidation of the scale. The use of the instrument will also provide opportunities for intercountry comparisons. The use of the instrument will also extend to longitudinal studies and be appropriate in providing baseline data for intervention research.

\section{Conclusion}

The index codes and their descriptions demonstrated face, content and construct validity with a high level of agreement. The inter- and intra-observer reliability for MIH and HSPM were "Substantial" to "Almost perfect". The present study confirms all the assumptions which were stated by previous studies which adds to the value of the index. The index demonstrated adequate evidence to be considered a valid and reliable tool for MIH/HSPM assessment in clinical practice and large epidemiological studies.

\section{Why this paper is important to paediatric dentists}

- Molar-Incisor Hypomineralisation (MIH) has many negative impacts on patients and presents diagnostic and treatment challenges to clinicians. A new assessment tool for measuring MIH/HSPM lesions was developed consistent with existing judgment criteria to grade the clinical conditions, amount of tooth surface area affected and other enamel defects.

- The study provides initial evidence of the reproducibility and validity of the MIH index to be considered a valid and reliable tool for MIH/HSPM assessment in clinical practice and large epidemiological studies.

- Future research using this index would provide additional information aimed at determining worldwide MIH prevalence.

\section{References}

1. Weerheijm KL, Jalevik B, Alaluusua S. Molar-Incisor Hypomineralisation. Caries Res 2001; 35: 390-391.

2. Zhao D, Dong B, Yu D, Ren Q, Sun Y. The prevalence of molar incisor hypomineralization: evidence from 70 studies. Int J Paediatr Dent 2018; 28:170-179.

3. Ghanim A, Morgan M, Mariño R, Bailey D, Manton D. Molar-Incisor Hypomineralisation: prevalence and defect characteristics in Iraqi children. Int $\mathbf{J}$ Paediatr Dent 2011; 21: 413-421.

4. Ghanim A, Manton D, Mariño R, Morgan M, Bailey D. Prevalence of demarcated 
hypomineralisation defects in second primary molars in Iraqi children. Int J Paediatr Dent 2013; 4: 110-113.

5. Elfrink MEC, Schuller AA, Weerheijm KL, Veerkamp JSJ. Hypomineralized Second Primary Molars: Prevalence Data in Dutch 5-Year-Olds. Caries Res 2008; 42: 282285 .

6. Jälevik B, Klingberg G. Treatment outcomes and dental anxiety in 18-year-olds with MIH, comparisons with healthy controls-a longitudinal study. Int J Paediatr Dent 2012; 22: 85-91.

7. Elfrink MEC, Ghanim A, Manton DJ, Weerheijm KL. Standardised studies on Molar Incisor Hypomineralisation (MIH) and Hypomineralised Second Primary Molars (HSPM): a need. Eur Arch Paediatr Dent 2015; 16: 247-255.

8. Jalevik B. Prevalence and Diagnosis of Molar-Incisor-Hypomineralisation (MIH): A systematic review. Eur Arch Paediatr Dent 2010; 11: 59-64.

9. Ghanim A, Elfrink MEC, Weerheijm KL, Mariño R, Manton DJ. A practical method for use in epidemiological studies on enamel hypomineralisation. Eur Arch Paediatr Dent 2015; 16: 235-246.

10. Ghanim A, Silva M, Elfrink M et al. Molar incisor hypomineralisation (MIH) training manual for clinical field surveys and practice. Eur Arch Paediatr Dent 2017; 18: 225242.

11. Owen ML, Ghanim A, Elsby D, Manton DJ. Hypomineralized second primary molars: prevalence, defect characteristics and relationship with dental caries in Melbourne preschool children. Aust Dent J 2018; 63: 72-80.

12. Gambetta-Tessini K, Mariňo R, Ghanim A, Calache H, Manton D. Carious lesion severity and demarcated hypomineralised lesions of tooth enamel in schoolchildren from Melbourne. Aust Dent J 2018; DOI: 10.1111/adj.12626.

13. Leen A. The interrelationship between molar hypomineralisation and orthodontics. [Thesis]. Melbourne: The University of Melbourne; 2013.

14. Lim JWH. Dental Caries and Molar Incisor Hypomineralisation (MIH): A prevalence study in an East Malaysian (Sabah) population. [Thesis]. Melbourne: The University of Melbourne; 2015.

15. Wang Y. Prevalence and presentation patterns of enamel hyomineralisation among paediatric hospital dental patients in Toronto, Canada. [Thesis]. The Hospital for Sick 
Children, Toronto, Canada; 2018.

16. Silva MJ, Kilpatrick N, Craig JM et al. Etiology of hypomineralized second primary molars: A prospective twin study. J Dent Res 2018; DOI: $10.1177 / 0022034516792870$.

17. Bolarinwa OA. Principles and methods of validity and reliability testing of questionnaires used in social and health science researches. Niger Postgrad Med J 2015; 22: 195-201.

18. Dawis RV. Scale construction. J Couns Psychol 1987; 34: 481-489.

19. Last J. A Dictionary of Epidemiology. 2nd. Edition. New York: Oxford University Press, Inc, 1988.

20. Silva MJ, Scurrah KJ, Craig JM, Manton DJ, Kilpatrick N. Etiology of molar incisor hypomineralization-A systematic review. Community Dent Oral Epidemiol 2016; 44: $342-353$.

21. Silva MJ, Kilpatrick N, Crombie F, Ghanim A, Manton DJ. What's new in Molar Incisor Hypomineralisation. Dent Update 2017; 44: 100-106.

22. Kimberlin CL, Winterstein AG. Validity and reliability of measurement instruments used in research. Am J Health Sys Pharm 2008; 65: 2276-2284.

23. Nunnally JC. Psychometric Theory. McGraw-Hill series in psychology, New York. 1987.

24. Murphy KR, Davidshofer CO. Psychological Testing: Principles and Applications Fifth Edition. Upper Saddle River: Prentice Hall, New Jersey. 2001.

25. Landis JR, Koch GG. The measurement of observer agreement for categorical data. Biometrics 1977; 33: 159-174.

26. World Health Organization. Calibration of examiners for oral health epidemiological survey. Geneve: The Oral Health Programme, WHO; 1993.

27. Elfrink ME, Veerkamp JS, Aartman IH, Moll HA, Ten Cate JM. Validity of scoring caries and primary molar hypomineralization (DMH) on intraoral photographs. Eur $\mathbf{J}$ Paediatr Dent 2009; 10 Suppl 1: 5-10.

28. MEDCALC. Free statistical calculators. Diagnostic test evaluation calculator https://www.medcalc.org/calc/diagnostic test.php Accessed July 4, 2018. 
29. Jasulaityte L, Weerheijm KL, Veerkamp JS. Prevalence of molar-incisorhypomineralisation among children participating in the Dutch National Epidemiological Survey (2003). Eur J Paediatr Dent 2008; 9: 218-223.

30. Elfrink ME, ten Cate JM, Jaddoe VW, Hofman A, Moll HA, Veerkamp JS. Deciduous molar hypomineralization and molar incisor hypomineralization. J Dent Res 2012; 91: 551-5.

31. Weerheijm KL, Duggal M, Mejàre I et al. Judgement criteria for molar incisor hypomineralisation (MIH) in epidemiologic studies: a summary of the European meeting on MIH held in Athens, 2003. Eur J Paediatr Dent 2003; 4: 110-3.

32. Brunette DM. Critical thinking: understanding and evaluating dental research. London: Quintessence Pub. Co.; 1996.

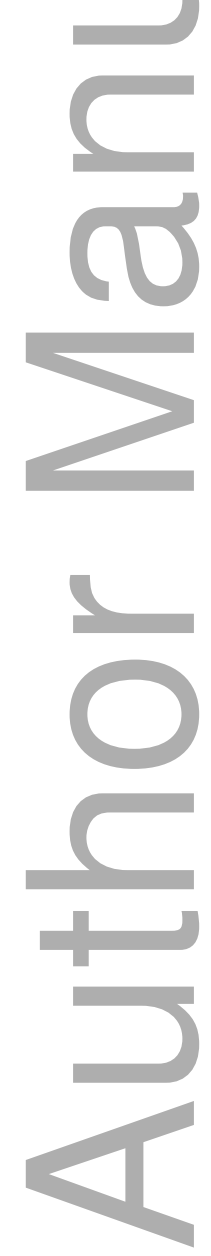


Table 1: Description criteria of MIH index.

\section{Clinical status criteria}

$0=$ No visible enamel defect.

$1=$ Enamel defect, not MIH/HSPM.

$2=$ White, creamy demarcated, yellow or brown demarcated opacities.

3 = Post-eruptive enamel breakdown (PEB).

$4=$ Atypical restoration.

$5=$ Atypical caries

$6=$ Missing due to MIH/HSPM.

$7=$ Cannot be scored.

Lesion extension criteria

$\mathrm{I}=$ less than one third of the tooth affected.

II $=$ at least one third but less than two thirds of the tooth affected.

III = at least two thirds of the tooth affected.

Modified after Ghanim et al., $2015^{9}$.

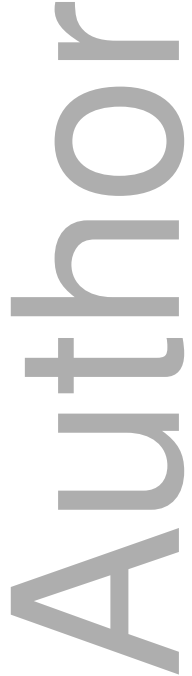


Table 2: Frequency distribution and mean number of caries affected teeth in relation to MIH lesion severity and extension

\begin{tabular}{|c|c}
\hline & lesion severity and extension \\
\hline Tooth status & Mean DMFT \pm SD \\
\hline Sound & $1.11 \pm 1.86$ \\
\hline Opacity only & $(314)$ \\
\hline Soumber of affected teeth with caries (Total N=424) \\
\hline Sound breakdown & $1.80 \pm 3.00$ \\
\hline
\end{tabular}

\begin{tabular}{|c|c|}
\hline MIH lesion extension \\
\hline$<1 / 3$ & $2.45 \pm 1.71$ \\
\hline$>1 / 3,<2 / 3$ & $(49)$ \\
\hline$>2 / 3$ & $4.00 \pm 2.49$ \\
\hline$>$ & $(35)$ \\
\hline
\end{tabular}

SD: standard deviation 


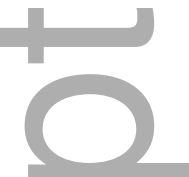

Table 3: Distribution of enamel breakdown and opacities in relation to the number of individuals with HSPM affected teeth

\begin{tabular}{|c|c|c|c|}
\hline & \multicolumn{3}{|c|}{ Defect type } \\
\hline & Creamy/white & Yellow/brown & Post-eruptive breakdown/ \\
\hline Number and $(\%)$ of & Opacities & Opacities & Atypical restoration \\
\hline $\begin{array}{l}\text { Participants with Three or } \\
\text { more HSPM affected teeth }\end{array}$ & $5(20.8)$ & $17(70.8)$ & $2(8.4)$ \\
\hline $\begin{array}{l}\text { Participants with Two HSPM } \\
\text { affected teeth } \\
19(21.6)\end{array}$ & $11(57.9)$ & $8(42.1)$ & -- \\
\hline $\begin{array}{l}\text { Participants with One HSPM } \\
\text { affected tooth } \\
45(51.1)\end{array}$ & $29(67.4)$ & $16(35.6)$ & -- \\
\hline Total $(\mathrm{n}=88)$ & $45(51.1)$ & $41(46.6)$ & $2(2.3)$ \\
\hline
\end{tabular}

HSPM: hypomineralised second primary molar. 


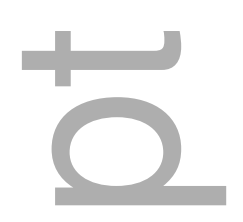

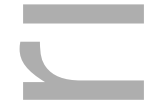

Table 4: Inter-examiner and test-retest reliabilities results.

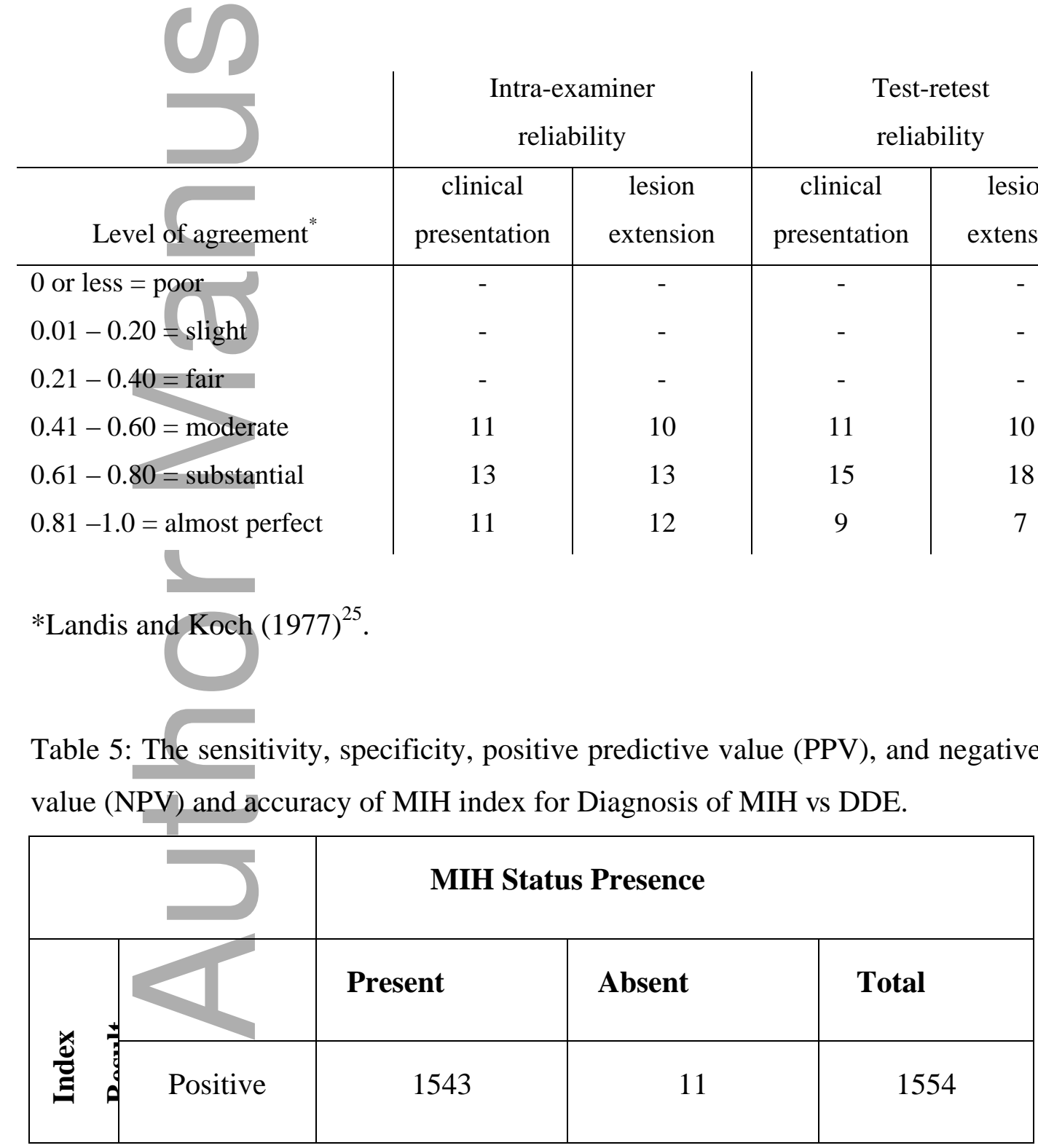




\begin{tabular}{|c|c|c|c|c|}
\hline \multicolumn{2}{|c|}{} & \multicolumn{2}{c|}{ MIH Status Presence } \\
\hline \multirow{2}{*}{$:$} & Present & Absent & Total \\
\cline { 2 - 5 } & $\begin{array}{c}\text { Negative } \\
\text { Total }\end{array}$ & 32 & 514 & 546 \\
\hline & 1575 & 525 & 2100 \\
\hline
\end{tabular}

\begin{tabular}{rcccc}
\hline Sensitivity & Specificity & $\begin{array}{c}\text { Positive predictive } \\
\text { Value }\end{array}$ & $\begin{array}{c}\text { Negative predictive } \\
\text { Value }\end{array}$ & Accuracy \\
$95 \%(\mathrm{CI})$ & $95 \%(\mathrm{CI})$ & $95 \%(\mathrm{CI})$ & $95 \%(\mathrm{CI})$ & \\
& $97 \%(\mathrm{CI})$ & \\
\hline $98.0 \%$ & $97.9 \%$ & $99.3 \%$ & $94.1 \%$ & $97.9 \%$ \\
$97.1-98.6 \%$ & $96.2-98.9 \%$ & $98.7-99.6 \%$ & $91.9-95.8$ & $97.3-98.5$ \\
\hline
\end{tabular}




\section{University Library}

\section{- M M I N E R VA A gateway to Melbourne's research publications}

Minerva Access is the Institutional Repository of The University of Melbourne

Author/s:

Ghanim, A;Marino, R;Manton, DJ

Title:

Validity and reproducibility testing of the Molar Incisor Hypomineralisation (MIH) Index

Date:

2019-01-01

Citation:

Ghanim, A., Marino, R. \& Manton, D. J. (2019). Validity and reproducibility testing of the Molar Incisor Hypomineralisation (MIH) Index. INTERNATIONAL JOURNAL OF PAEDIATRIC DENTISTRY, 29 (1), pp.6-13. https://doi.org/10.1111/ipd.12433.

Persistent Link:

http://hdl.handle.net/11343/284668 